\title{
Extracorporeal Membrane Oxygenation (ECMO): An Option for Cardiac Reccovery from Advanced Cardiogenic Shock
}

\author{
Ali Ghodsizad MD, PhD,${ }^{1}$ Kai Singbartl, MD,${ }^{2}$ Matthias Loebe, MD,${ }^{1}$ Mohamed Zeriouh MD, ${ }^{3}$ \\ Arjang Ruhparwar MD, ${ }^{3} \mathrm{PhD}$, April Grant, MD, ${ }^{1}$ Ali El-Banayosy MD, ${ }^{2}$ M.M. Koerner MD, $\mathrm{PhD}^{2}$ \\ ${ }^{1}$ Miami Transplant Institute, University of Miami, Jackson Memorial Hospital, Miami, Florida, USA; ${ }^{2}$ Heart and Vascular \\ Institute, Penn State Milton S. Hershey Medical Center, Penn State College of Medicine, Hershey, Pennsylvania, USA; \\ ${ }^{3}$ Department of Surgery, University of Heidelberg, Germany
}

\section{ABSTRACT}

Mechanical circulatory support can prevent multi-organ failure and death in patients with advanced cardiogenic shock. Here we describe our experience using extracorporeal membrane oxygenation (ECMO) for treatment of advanced cardiogenic shock which has been used by our team for daily routine care in more than 200 patients during the last five years at the Penn State Medical Center.

Venoarterial (VA) ECMO has been used as a viable therapeutic option for advanced cardiogenic shock as a bridge to recovery (BTR) or bridge to next decision (BTD). Our group performed a retrospective review of data from 155 patients from our single center cohort treated with VA ECMO for advanced cardiogenic shock. After successful ECMO treatment, the one year survival rate of patients with ischemic heart disease was $73.7 \%$, and the one year survival for patients with non-ischemic heart disease was $75 \%$.

\section{INTRODUCTION}

Despite the widespread employment of early revascularization, patients with advanced cardiogenic shock still have high morbidity and mortality rates. Short-term mechanical circulatory support using extracorporeal membrane oxygenation (ECMO) has been successfully used to treat cardiogenic shock. Various devices, including the Tandem Heart ${ }^{\circledR}$, Impella Microaxial Pump ${ }^{\circledR}$ and other well-known rescue pumps have been used for this purpose. Here we will discuss the management of advanced cardiogenic shock using venoarterial (VA) ECMO.

In 1885 Gruber and colleagues developed the first oxygenator/artificial lung [Lim 2006] MC Lean and colleagues [McLean 1959] discovered heparin in 1916, which was essential for development of extracorporal technology and later for the application of ECMO. A further important milestone in the development of the extracorporal circulation, was the use of a roller pump by Dr. Michael Debakey in Houston, Texas in 1932. The first report of a successful clinical application of

Received fune 21, 2016; received in revised form fuly 25, 2016; accepted August 2, 2016.

Correspondence: Dr. A. Ghodsizad, Department of Cardiac Surgery, Heart and Vascular Institute, Penn State Milton S. Hershey Med Center, Penn State College of Medicine, 500 University Dr, Hershey, PA 17033; (e-mail:aghodsi@gmx.org).
ECMO for the treatment of acute respiratory distress syndrome (ARDS) was presented by Hill et al in 1970. Initially discouraging and promoting results were published by different groups, depending on the characteristics of treated patients. Some studies did not show any improvement of mortality, while recently others showed a survival benefit in patients, who received ECMO implantation [Zapol 1979, Peek, 2010]. Patients with cardiogenic shock have critically low survival rates. Different non randomized publications have shown mixed results, including low to high survival benefits $(25-70 \%)$ in patients with cardiogenic shock after veno-arterial ECMO application [Muehrcke 1996, Luo 2009]. Here we are describing our experience with an advanced ECMO circuit, which has been used by our team for daily routine care in more than 200 patients during the last 5 years at Penn State Medical Center.

\section{Intention to treat}

The intention to treat is either bridge to recovery (BTR) or bridge to next decision (BTD). Every effort should be made to enhance and optimize myocardial recovery. In cases of insufficient myocardial recovery, the patient should be evaluated for a long term assist device or a total artificial heart. In cases of irreversible severe brain damage or ineligibility for long-term MCS, the patient should be evaluated for organ donation or an end-of-life decision.

Table 1. The primary causes of advanced cardiogenic shock

Etiology

Acute myocardial infarction

Fulminant myocarditis

Postcardiotomy cardiogenic shock

Post cardiac transplantation (primary graft failure, late rejection)

Acute decompensated chronic heart failure/dilated cardiomyopathy

Postpartum cardiomyopathy

Hypertrophic non-obstructive cardiomyopathy

Drug intoxication

Hypothermia

Septic shock

Hypoxia due to respiratory failure 

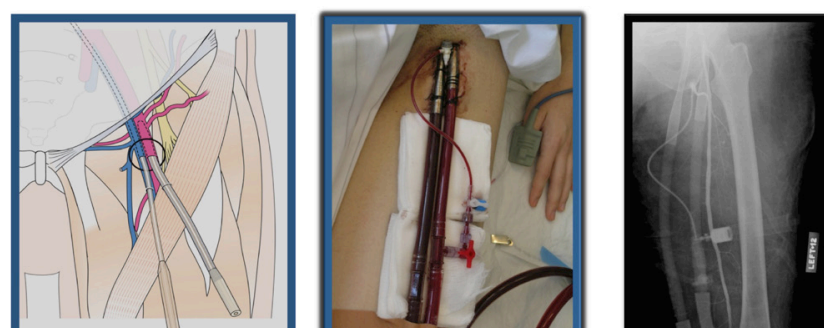

Figure 1. A, B. (Sketch and photographs) Inserted venous and arterial cannulas in the left groin and the antegrade perfusion of the lower limb. C, Bedside fluoroscopy showing correct placement of the perfusion sheath in the superficial femoral artery. *

Table 2. Primary contraindications to ECMO

Contraindications

Existing disease with expected mortality rate of $>95 \%$

Intolerance to anticoagulation

Aortic dissection

Do not resuscitate (DNR) orders

End stage heart failure not amenable to VAD implantation and/or transplantation

Irreversible neurologic damage

Relative contraindications

Overwhelming proven sepsis

Intracerebral hemorrhage

Aortic insufficiency

Severe immunosuppression (ANC <400)

Advanced age

Severe peripherial vascular disease

Active life-limiting disease

\section{Indications}

Advanced cardiogenic shock as a result of different causes is the main indication for venoarterial ECMO. A full understanding of the complexity of disease in this patient group entails assessment of criteria for shock: hypotension, (SBP $<90 \mathrm{mmHg}$ ) evidence for end-organ hypoperfusion, heart rate of $\geq 60$ beats per minute, a cardiac index of no greater than 2.2 liters per minute and a PCWP of $15 \mathrm{~mm} \mathrm{Hg}$ [Hochman 1999] A clear indication for ECMO are patients with advanced cardiogenic shock, which includes a systolic blood pressure $<90 \mathrm{mmHG}$ despite 2 or more i.v. inotropes with or without IABP, evidence of decreased organ perfusion, PCWP $>18 \mathrm{mHG}$ and a $\mathrm{CI}<2.1 \mathrm{~L} / \mathrm{min} / \mathrm{m}$. The etiology for cardiogenic shock can be acute myocardial infarction, postcardiotomy shock, and acute myocarditis and other causes (Table 1).

\section{Contraindications}

Contraindications to ECMO include: intolerance to anticoagulation; aortic dissection; do not resuscitate (DNR)
Table 3. Parameters for monitoring and assessment of LV unloading

Monitoring of LV unloading

Keep the diastolic pulmonal artery pressure $<25 \mathrm{mmHG}$

Maintain the LV ejection above ECMO, using inotropes and adjustment of ECMO flow

Reduce volume overload

Methods

Intra Aortic Balloon Pulsation (IABP)

Percutaneous pulmonal artery drain catheter

Impella Microaxial Pump

Septostomy

PulseCath ${ }^{\circledR}$ Heart circulatory support system (not available in USA)

orders; end stage heart failure not amenable to VAD implantation and/or transplantation; irreversible neurologic damage; and comorbidity with non-cardiac disesase with high expected mortality rates. In borderline cases, the decision to institute ECMO should be based on a multidisciplinary approach using a case-by-case management strategy (Table 2).

\section{The ECMO Circuit}

Historically, the first ECMO circuits utilized roller pumps or early generation centrifugal pumps, basic oxygenators, and long tubing sets. Cannulation techniques used an invasive surgical approach with cut-down. The associated surgical trauma and bleeding and were the major disadvantages of these early ECMO systems making them suitable for only short-term support.

Currently we use a new generation of simplified ECMO circuits, which consists of a low pressure drop oxygenator, a magnetically levitated centrifugal pump, and a simplified Carmeda ${ }^{\circledR}$ coated tubing system. Modifications in pump design, bearings, rotation seals, and others miminize shear stress which reduces pump thrombosis, thus lowering the risk of hemolysis and clot formation. These allow us to run ECMO with a lower level of anticoagulation, minimizing the potential for adverse events (Figure 1). The Carmeda ${ }^{\circledR}$ coated tubing set also can effectively help prevent clot formation. The use of a shorter tubing circuit minimizes the biological interface between the blood and artificial surface, which reduces the risk of complement activation, coagulation deficiencies, and activation of inflammatory pathways which could result in systemic inflammatory response syndrome (SIRS). This new circuit design also enables safer patient transfer for diagnostic measures in the hospital and safer transfer between hospitals, even for long distances, including intercontinental transfer (Figure 4).

\section{Cannulation techniques}

A variety of different cannulation techniques can be used for initiation of the ECMO support. ECMO is established by direct cannulation of the right atrium and ascending aorta. The placement of the so-called central ECMO enables higher pump flows due to use of a larger cannula size. Although 

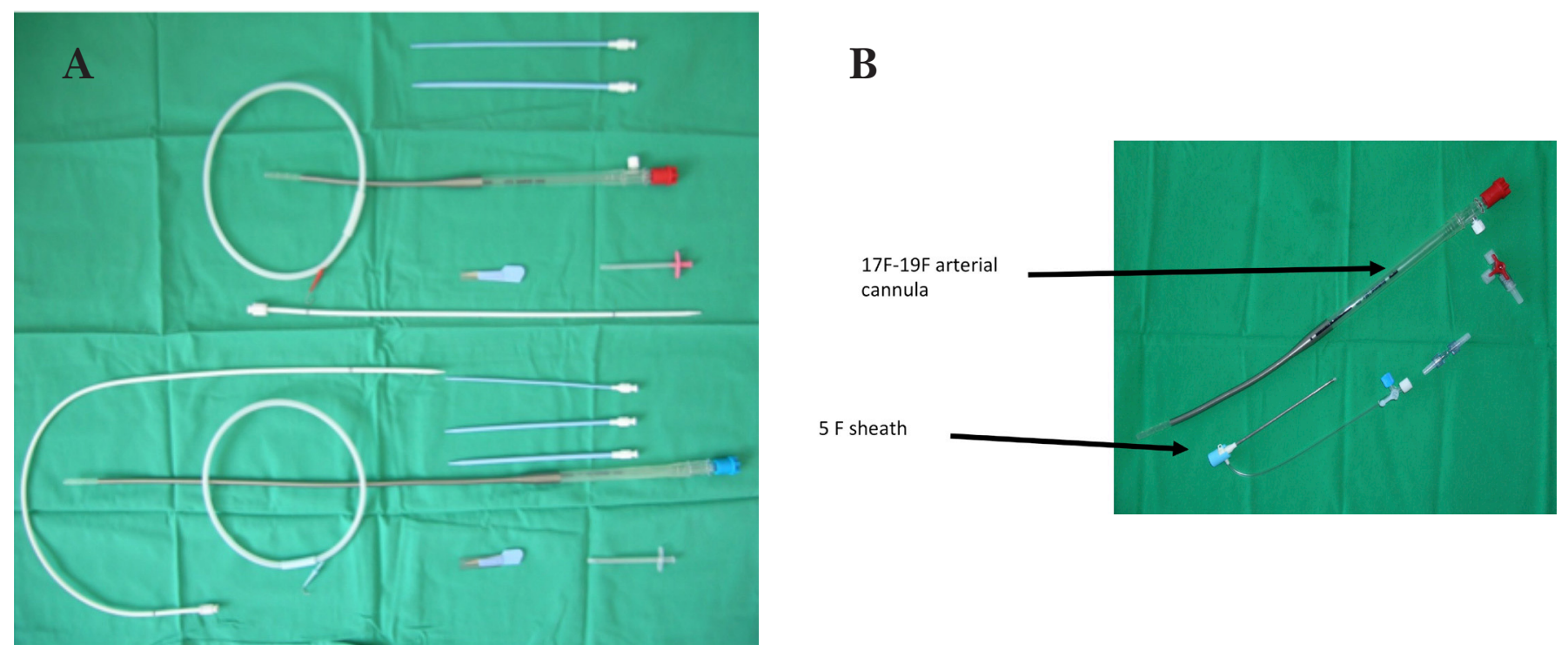

Figure 2. A, Percutaneous cannulation set used for ECMO cannulation. B, Arterial cannula and sheath.*

central ECMO allows for better drainage of the left ventricle, it requires a surgical approach. After sternotomy and dissection of the pericardium, the venous and arterial cannulas are placed, and venting of the left atrium can be started. Surgical implantation requires adequate anticoagulation which increases the risk of major bleeding in patients on I.V. heparin and ECMO support. Patients are also at higher risk of infection and other postoperative complications. For these and other reasons, we prefer to use peripherial cannulation.

We use the Carmeda ${ }^{\circledR}$ coated flat wound cannula (BioMedicus ${ }^{\circledR}$ Femoral Venous and Arterial Cannula), which is available in 15-21 Fr and allows cautious heparinization. The common femoral artery and vein can be used as alternative sites for establishment of extracorporeal cardiopulmonary support. A serious complication of prolonged femoral cannulation is ischemic injury of the cannulated distal limb. To minimize this, we maintain antegrade femoral blood flow by positioning a vascular introducer percutaneously distal to the arterial ECMO cannula into the superficial femoral artery, entering the femoral vessels distally to the inguinal ligament. The antegrade limb perfusion sheath using 5-7 Fr sheath for the perfusion of the distal limb is connected into the side port of the arterial line. Fluoroscopy is used to confirm the correct placement of the antegrade limb perfusion sheath. We have found this technique to be safe and effective in preventing lower limb ischemia for patients with prolonged femoral cannulation for extracorporeal circulatory support. Puncturing the vessels and advancement of the guidewire, to dilate and advance the cannula into the vessel can be more challenging in obese patients and patients with extensive peripherial vascular disease. In these cases, the axillary vein and artery in the deltopectoral fossa offer an alternative access point for cannulation. Adequate limb perfusion in our patients is monitored using a noninvasive somatic oxymeter (Adult SomaSensors; Somanetics $^{\circledR}$, Covidien, Mechanicsburg, PA, USA) to detect regional hemoglobin oxygenation saturation.

\section{Antioagulation management}

All patients receive $100-120$ IE heparin $/ \mathrm{kg}$ by weight before cannulation, unless they were previously on antithrombotic therapy or in postcardiotomy shock. We maintain anticoagulation with continuous intravenous heparin to maintain partial thromboplastin time (PTT) levels of 50-60 seconds. Pump flows below $2.5 \mathrm{~L} / \mathrm{min}$ a PTT level of between 60-80 seconds are required for weaning. In patients with severe bleeding disorders, heparin can be completely stopped. Heparin-induced thrombocytopenia (HIT) predisposes to thrombosis and clot formation. Screening for HIT in patients on ECMO is a necessary, and the presence of HIT is an indication to switch to an alternative anticogulation medication, such as I.V. Agatroban.

\section{Hemodynamic management}

As part of the pre-implantation diagnostics, a pulmonary artery catheter is used to monitor pulmonary artery pressure, mixed venous saturation, central venous pressure and pulmonary capillary wedge pressure (PCWP). The majority of these patients are grossly volume overloaded. Every effort should be made to achieve an euvolemic status, including using diuretics and continuous renal replacement therapy (CRRT). Monitoring of systolic, diastolic and mean arterial pressure is also important. Following the institution of ECMO, inotropes, vasopressors and volume therapy are used and titrated to maintain an ECMO flow greater than $4 \mathrm{~L} / \mathrm{min}$, pulmonal artery end diastolic pressures of $<24-25 \mathrm{mmHg}, \mathrm{MAP}$ $>$ than $60 \mathrm{~mm} \mathrm{Hg}$, and mixed venous saturation $>60 \%$. The overall goal is to achieve adequate organ perfusion while maintaining adequate left ventricular unloading. The right radial artery should be used for invasive arterial blood pressure monitoring, and the punture site for arterial blood gas sampling.

\section{Left ventricular unloading}

In contrast to other percutaneous short term support devices, VA ECMO can provide adequate right heart and pulmonary 


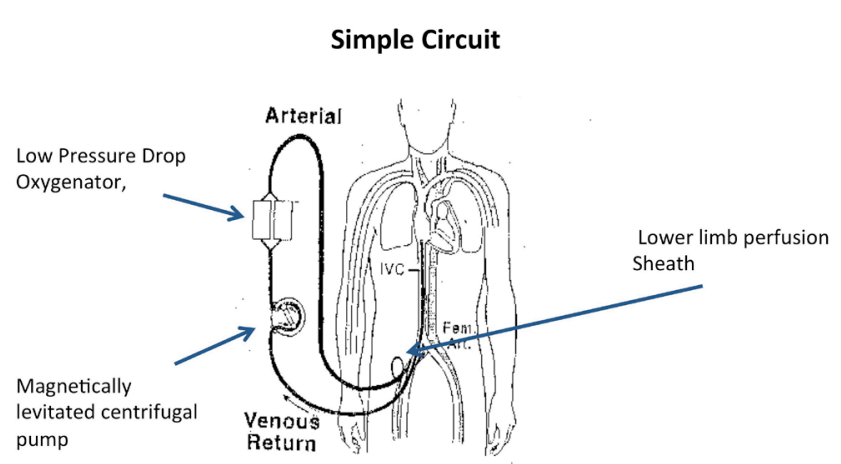

Figure 3. Sketch showing basic ECMO circuit and connection to the patient.*

support. However, one of the major challenges and disadvantages of VA ECMO is incomplete $\mathrm{LV}$ unloading, which is seen in about $10 \%$ of all veno-arterial cases in our experience. To minimize the risk of pulmonary edema and inadequate $\mathrm{LV}$ unloading, we employ aggressive diuretic therapy, titration of vasopressors and inotropes, adjustment of ECMO flows, and daily assessment using echocardiography (Table 3). In cases where conservative management is not sufficient, we perform a septostomy as the preferred invasive method.

\section{Weaning}

Monitoring of the weaning process is done using hemodynamic measurements and daily transthoracic and/or transesophageal echocardiograms (TTE or TEE). Patient hemodynamics should be recorded on full and partial (50\%) pump support. When a $50 \%$ reduction is achieved, if the LV shows signs of recovery (maintaining mean arterial pressure (MAP), left ventricular ejection above ECMO flow, and LVEF > $30 \%$ ), a bolus of heparin is given and the pump should be stopped. With the pump turned off, echocardiograms and hemodynamic measurements should be repeated. The patient is considered weanable when the following criteria are fulfilled: absent volume overload with minimal inotropic support ( $<5 \mathrm{ug} / \mathrm{kg}$ of dopamine or the equivalent), left ventricular ejection fraction (LVEF) greater than $30 \%$, cardiac index of $2.4 \mathrm{~L} /$ $\mathrm{min} / \mathrm{m}^{2}$, MAP greater than $60 \mathrm{~mm} \mathrm{Hg}$, pulmonary capillary wedge pressure (PCWP) less than $18 \mathrm{mmHG}$ and a central venous pressure (CVP) less than $18 \mathrm{~mm} \mathrm{Hg}$.

\section{End-of-life decision making}

For patients unable to be weaned from ECMO and who are not eligible for long-term mechanical circulatory support or organ donation, it is reasonable to consider them for an end-of-life decision. This can be made in conference with the family, social workers, palliative care representatives, VAD coordinators, nursing staff, nurse practitioners, chaplains, and in borderline cases, with the institutional ethics committee.

\section{DISCUSSION}

Our group performed a retrospective review of 155 patients from our single center cohort treated with VA ECMO for

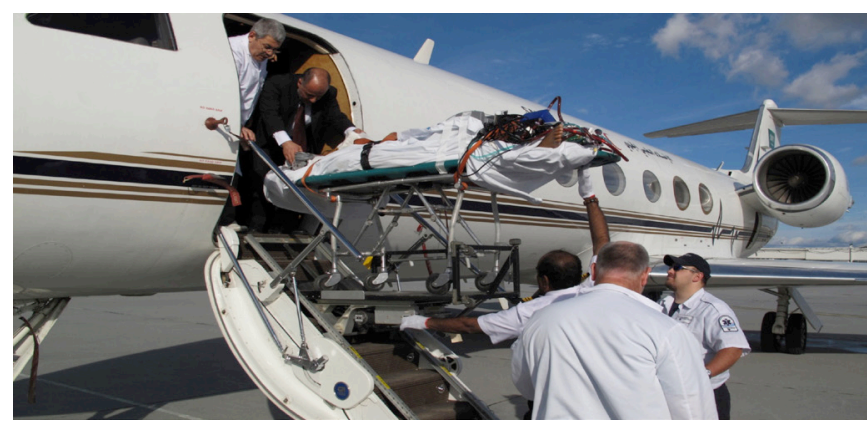

Figure 4. Intercontinental patient transfer on ECMO support.*

advanced cardiogenic shock [Pabst 2016]. Out of 155 total patients, $83 \%$ were successfully weaned from ECMO and discharged from the hospital. Forty-three $(51.8 \%)$ of the patients had developed cardiogenic shock due to ischemic heart disease. Thiry-four (30.1\%) had postcardiotomy cardiogenic shock, which included 15 patients with postcardiotomy shock following sole or concomitant myocardial revascularization. After successful ECMO treatment, the one year survival rate of patients with ischemic heart disease was $73.7 \%$, and the one year survival for patients with non-ischemic heart disease was $75 \%$.

\section{ACKNOWLEDGEMENTS}

We thank Drs. C Brehm and D Pabst for their kind support.

*A Ghodsizad, A El-Banayosy, C Brehm, MM Koerner, Book chapter: Cardiac Surgery; Technique and Practice, Chapter 155; Acute mechanical cardiac support/ LVAD: ECMO (veno-arterial), M. Slaughter (Ed), McGraw-Hill Professional, New York (in press)

\section{REFERENCES}

Hochman JS et al. 1999. Early revascularization in acute myocardial infarction complicated by cardiogenic shock. SHOCK Investigators. Should We Emergently Revascularize Occluded Coronaries for Cardiogenic Shock. N Engl J Med 341:625-634.

Lim MW. 2006. The history of extracorporeal oxygenators. Anaesthesia 61:984-995.

Luo XJ, Wang W, Hu SS et al. 2009. Extracorporeal membrane oxygenation for treatment of cardiac failure in adult patients. Interact Cardiovasc Thorac Surg 9:296-300.

McLean J. 1959. The discovery of heparin. Circulation 19:75-78.

Muehrcke DD, McCarthy PM, Stewart RW et al. 1996. Extracorporeal membrane oxygenation for postcardiotomy cardiogenic shock. Ann Thorac Surg 61:684-691.

Pabst D, Ghodsizad A, El-Banayosy et al. 2016. Successfully weaned patients after VA ECMO due to cardiogenic shock: Etiology and 1-year survival. Jnl of Heart and Lung Transplantation 35:4, S372.

Peek GJ, Elbourne D, Mugford M et al. 2010. Randomised controlled trial and parallel economic evaluation of conventional ventilatory support versus extracorporeal membrane oxygenation for severe adult respiratory failure (CESAR). Health Technol Assess 14:1-46. 